\title{
An Overview of Spray Modeling With OpenNCC and its Application to Emissions Predictions of a LDI Combustor at High Pressure
}

\author{
M.S. Raju* \\ Vantage Partners LLC (NASA GRC GESS-3 Contract) \\ 3000 aerospace parkway \\ Brookpark, Ohio-44132
}

\begin{abstract}
The open national combustion code (OpenNCC) is developed with the aim of advancing the current multi-dimensional computational tools used in the design of advanced technology combustors. In this paper we provide an overview of the spray module, LSPRAY-V, developed as a part of this effort. The spray solver is mainly designed to predict the flow, thermal, and transport properties of a rapidly evaporating multi-component liquid spray. The modeling approach is applicable over a widerange of evaporating conditions (normal, superheat, and supercritical). The modeling approach is based on several well-established atomization, vaporization, and wall/droplet impingement models. It facilitates large-scale combustor computations through the use of massively parallel computers with the ability to perform the computations on either structured \& unstructured grids. The spray module has a multi-liquid and multi-injector capability, and can be used in the calculation of both steady and unsteady computations. We conclude the paper by providing the results for a reacting spray generated by a single injector element with $60^{\circ}$ axially swept swirler vanes. It is a configuration based on the next-generation lean-direct injection (LDI) combustor concept. The results include comparisons for both combustor exit temperature and $E I N O_{X}$ at three different fuel/air ratios.
\end{abstract}

\section{INTRODUCTION}

In an effort to guide in the design and testing of advanced gas-turbine combustors, NASA Glenn research center (GRC) has undertaken the development

\footnotetext{
*AIAA associate fellow.
}

of OpenNCC. Since its inception about 20 years ago, the code has gone though considerable evolution to accommodate the changing needs of various ongoing projects associated with next-generation combustor technology development. More on the current status of OpenNCC can be found in Refs. [1 \& 2]. There are many aspects to the development of OpenNCC but in this paper we describe some important features of the spray module [3].

There are many occurrences of sprays in a variety of industrial and power applications and materials processing [4]. A liquid spray is a two phase flow with the gas as the continuous phase and the liquid as the dispersed phase in the form of droplets or ligaments [4]. The coupling between the two phases occurs through the exchanges of mass, momentum, and energy involving a wide range of thermal, mass, and fluid dynamic factors. A number of finite-difference formulations have been advanced over the years for predicting the flow (mass and momentum) and thermal properties of a rapidly vaporizing spray. Some of the pros and cons of various formulations can be found in [4-8].

In this paper, we summarize some important aspects of our spray formulation without making any attempt to provide an in-depth review on the fluid dynamic and transport behavior of reacting sprays [9-18]. Depending on the nature of the spray, an appropriate selection could be made from the choice of various well-known spray formulations (multicontinua, discrete-particle, or probabilistic) based on either a Lagrangian or an Eulerian representation for the liquid-phase equations by making use of appropriate droplet sub-grid models. The present solution procedure could be used within the context of both 
multicontinua and probabilistic spray formulations, as it allows for resolution on a scale greater than the average spacing between two neighboring droplets [4]. For OpenNCC, the adopted choice for the gas phase was an Eulerian scheme. The liquid-phase equations form a system of hyperbolic equations and they could be solved by means of either an Eulerian or a Lagrangian representation. A Lagrangian scheme is chosen as it reduces the errors associated with numerical diffusion. The liquid-phase formulation is based on various well-established models for droplet drag; the vaporization models of a polydisperse spray take into account the transient effects associated with the droplet internal heating and the forced convection effects associated with droplet internal circulation; and it employs models for gas-film valid over a wide range of low to intermediate droplet Reynolds numbers [10]. Our current formulation is applicable for flows with a dilute spray approximation where the droplet loading is low. The numerical method could be used within the context of both steady and unsteady calculations [11-18]. Not considered in the present release of the code are the effects associated with droplet/shock interaction and dense spray effects. Some major features of the spray module are summarized as follows:

- It facilitates the use of both structured \& unstructured grids and parallel computing and, thereby, facilitates large-scale combustor computations involving complex geometrical configurations. The solver accommodates the use of an unstructured mesh with mixed elements of either triangular, quadrilateral, and/or tetrahedral type [14].

- In order to deal with modern gas-turbine fuels that are mixtures of many compounds, it takes into account the modeling of multicomponent liquid fuels with variable properties [3].

- Various well-established vaporization and atomization models are incorporated into our spray code to cover a wide range of engine operating conditions from low to high pressures as well as flash vaporization associated with superheat conditions [3]. The initial droplet conditions could be prescribed based on either a single-point or multi-point droplet injection. The multi-point injection could be in the form of a line or circular point injection. The initial droplet conditions could be specified entirely in the form of a table based on known experimental data, or some of the initial conditions could be calculated based on several widely-used droplet-size distribution functions or primary atomization models incorporated into our spray code. For low pressures, we have incorporated the following primary atomization models: (a) sheet breakup, (b) blob jet, or (c) BLS (Boundary-Layer Stripping) [3]. We have also incorporated a flash induced atomization model to cover superheat conditions. Any further droplet breakup after primary atomization could be modeled based on the following set of secondary droplet breakup models: (a) Rayleigh-Taylor, (b) TAB (Taylor Analogy Breakup), or (c) ETAB (Enhanced Taylor Analogy Breakup) [3].

- Our spray code supports all of the boundary conditions of the national combustion code including particle movement through very complex periodic boundary conditions. Upon impact with the wall, a droplet may shatter, rebound, or stick to the wall depending on the level of collision impact. We have implemented several droplet-wall interaction models into our spray code to cover a wide range of conditions.

- The spray module is designed in such a way so that it could easily be coupled with other CFD codes.

With the aim of improving the overall solution procedure of the national combustion code involving sprays, we have made several other relevant contributions to the gas-side of computations:

- In order to demonstrate the importance of chemistry/turbulence interactions in the modeling of reacting sprays, we have extended the joint scalar Monte Carlo PDF (Probability Density Function) approach to the modeling of spray flames, unstructured grids, and parallel computing [14 \& 19].

The spray solution procedure provided favorable results when applied to the modeling of several reacting/non-reacting sprays [1-2, 11-18, \& 21-22].

One of the major driving factors behind the development efforts of next-generation combustor design at NASA GRC is the need to meet the nextgeneration gas-turbine combustor emissions targets set for $N O_{X}$ reduction [20]. More specifically, this effort was funded through several NASA projects 
including ERA (Environmentally Responsible Aviation) [21]. The combustor design based on the LDI concept has received considerable attention as it offered promising results in emissions reduction. It is essentially based on a multipoint fuel-injection \& multi-lean-burning-zone concept with all the air entering through the dome with no dilution; each injector has an air-swirler to provide rapid mixing for burning; \& the LDI design leads to the formation of small lean-burning zones to promote low NOx [20]. There are currently several experimental studies underway to evaluate its feasibility of achieving both higher fuel efficiency and lower combustion emissions over a wide range of combustor operating conditions [22]. Also, several CFD studies were undertaken to evaluate engine designs based on the LDI concept $[2,17-19, \& 21-22]$. They included results from both single and multi-element LDI injectors based on OpenNCC RANS (Reynolds averaged Navier Stokes) $[2,17-18, \&$ 21-22], URANS (unsteady RANS) [2 \& 21], very large eddy simulation based on TFNS (time filtered Navier Stokes) [2, \& 21], \& the combined Monte-Carlo PDF/spray/CFD approach [21]. More recently, Ajmani et al [21] reported assessment of OpenNCC RANS in emissions predictions for both single and multi-element LDI-1 injectors based on the Woodward FT. The LDI-1 injectors were developed as a part of the next-generation emissions targets for $N O_{X}$ reduction set by NASA's N+1 ERA program.

One important aspect of spray modeling missing from the previous calculations is the lack of proper models valid for droplet vaporization at supercritical conditions. To overcome this deficiency, we have recently incorporated a high-pressure droplet vaporization model into our spray formulation [3] based on some important aspects of the modeling approach derived from [23-28]. As a part of this effort, we also implemented the Peng-Robinson equation of state following the approach of [29-31], the high-pressure corrections to the gas-phase transport properties from [29-30, \& 33-36], and the high-pressure corrections to the liquid-phase transport properties from $[29-30, \&$ $32]$.

In this paper we are going to revisit the investigation of a single-element LDI-1 injector [21] by making use of the newly implemented high-pressure droplet vaporization model. Actualy, this injector configuration was a derived from a more complex LDI combustor based on a 9-point swirl-Venturi injector. This simplification allows us to evaluate in great detail various physical models embedded into Open-
NCC. More importantly, Ajmani et al [21-22] used this configuration to assess the accuracy of some reduced chemical-kinetic mechanisms developed to improve the current capabiity of emissions predictions with OpenNCC. The present calculations are based on OpenNCC with the following options: RANS with standard two-equation turbulence model for gasphase, a Lagrangian spray model, the Peng-Robinson EOS (equation of state), \& a 14 -species \& 18-step reduced chemical kinetic mechanism.

\section{OVERVIEW OF LSPRAY-V}

\subsection{MULTI-COMPONENT LIQUID SPRAY MODELING}

It is well known that most of the gas-turbine fuels are multicomponent mixtures of many compounds with a wide distillation curve. The multicomponent nature of the liquid sprays is more evident with the increasing need to use jet fuels derived from heavier petroleum compounds. The gasification behavior of a multicomponent fuel droplet may differ significantly over that of a pure single component fuel droplet. Also, the calculation of the variable thermo-transport properties of the liquid-mixtures becomes more important at high pressures. The flame ignition characteristics (such as the phenomena associated with flame blow-off and extinction conditions) could also be influenced by the non-uniform concentration of the fuels with different volatilities. With this in mind, we have implemented a spray formulation based on our earlier work [10]. This implementation was later modified to take into account the effect of variable liquid properties [3].

\subsection{EXTENSION TO LARGE-SCALE COMBUSTOR CALCULATIONS BASED ON THE COMBINED \\ CFD/SPRAY/MONTE-CARLO-PDF METHOD, PARALLEL COMPUTING, \& UNSTRUCTUTERED GRIDS}

With the aim of advancing the multidimensional computational tools used in the design of advanced technology combustors, we extended the spray computations to unstructured grids with the ability to run on massively parallel computers. This effort was undertaken within the context of developing a modeling approach based on the combined CFD/spray/scalar-Monte-Carlo-PDF calculations. In this approach, the mean gas-phase velocity 
and turbulence fields were determined from the solution of a conventional CFD method, the scalar fields of species and enthalpy from a modeled PDF transport equation using a Monte Carlo method, and a Lagrangian-based spray solver is used for the liquidphase representation. As part of this effort, we have advanced the spray computations in a number of important ways: (1) extension of the spray computations to unstructured grids, (2) extension of the Monte Carlo PDF approach to spray computations, and (3) demonstrating the feasibility of large scale combustor computations by developing and implementing an efficient parallel computing strategy. Further details of this modeling approach to turbulent reacting spray flows wwere described in [14]. Some results from the application of this modeling approach were summarized in Refs. [13-16].

\subsection{ATOMIZATIONS MODELING}

The success of any spray calculation depends a great deal on the ability to specify appropriate initial spray conditions. In order to reduce uncertainty associated with the specification of the initial droplet conditions, we have undertaken a validation effort to establish accuracy of various atomization models used in spray calculations.

Atomization refers to a process of the liquid jet breakup into droplets. There are many processes associated with the liquid jet breakup. In the inner nozzle flow, several factors (such as injector type, geometry, and size) influence the conditions at the injector exit (such as the velocity, the initial sheet or jet thickness, and the angle of droplet dispersion). One way to specify the initial spray conditions at the injector exit is to rely on the widely-used correlations. For a better description, a more accurate analysis is needed which takes into account the physics associated with inside bubble growth, cavity formation, and internal turbulence. Once a liquid exits outside of the nozzle, it becomes unstable under the influence of aerodynamic instabilities and finally breaks up into droplets. The widely known aerodynamic instabilities are of Rayleigh-Taylor and Kelvin-Helmholtz kind [37-39]. The Rayleigh-Taylor instability is due to inertia of the denser fluid opposing the system acceleration in a direction perpendicular to the interface of the denser fluid and the Kelvin-Helmholtz instability is caused by the viscous forces due to the relative motion of the fluids [38-39]. When the maximum amplitude of the most unstable wave reaches a critical value, some liquid is stripped of the main liquid core in the form of primary droplets. These droplets may further breakup into smaller droplets due to a process known as the secondary droplet breakup mechanism.

Based on a linear instability analysis of a $2 \mathrm{D}$ viscous incompressible fluid moving thorough an inviscid incompressible gas, Reitz and Bracco [37] characterized the break-up regimes to be four-fold: (1) Rayleigh breakup, (2) first wind-induced breakup, (3) second wind-induced breakup, (4) atomization. In the first two regimes, drops of sizes greater than or equal to the nozzle diameter are produced at distances far from the nozzle exit. In the applications of our interest, the last two regimes are more important where droplets much smaller than the nozzle diameter are produced near the nozzle exit. The knowledge gained from the instability analysis of various kinds [37, \& 40-41] is combined with some experimental observations to form the basis for various models developed for both primary atomization \& secondary droplet breakup. In this approach, the jet breakup is modeled first by making use of a drop representation approach in which discrete parcels of liquid are injected in the form of blobs with a characteristic size representative of the nozzle diameter instead of tracking an actual intact liquid core that forms at the nozzle exit. In the case of a planar or conical liquid sheet, the discrete parcels essentially represent liquid ligaments. Before the jet breakup the discrete parcels stay inside of the liquid core or sheet but after the jet breakup they move independently. The breakup criterion, atomization rate, drop size and velocity and the location of the newly formed droplets are primarily determined based on an instability analysis derived from the conservation equations of mass, momentum and energy. The analysis of the jet or sheet breakup into ligaments or droplets, the stripping of the liquid into fragments or droplets, and the formation of more droplets from further breakup of ligaments or fragments are all described under the classification of primary atomization.

Some of the large droplets that are formed immediately after the primary liquid jet breakup may further breakup into smaller droplets under the influence of aerodynamic instabilities. The large droplets first tend to flatten under the influence of aerodynamic pressure. Then large amplitude long wavelength waves caused by drop deceleration induce a Rayleigh-Taylor instability on the flattened drop causing it to breakup further into several relatively large-size product droplets. While at the same time short surface waves induce a Kelvin-Helmholtz insta- 
bility on the windward side of the parent drop resulting in the generation of much smaller product droplets. The breakup of the larger droplets into smaller droplets is described under the classification of secondary droplet breakup.

Most of the coding required for both the primary atomization and secondary droplet breakup models was provided by CFDRC. The CFDRC atomization module contains the following four primary atomization models: (1) the sheet breakup primary atomization model, (2) the blob jet primary atomization model, \& (3) the BLS (BoundaryLayer Stripping) primary atomization model, and it also contains the following three secondary droplet breakup models: (1) the Rayleigh-Taylor secondary droplet breakup model, (2) the TAB (Taylor Analogy Breakup) secondary droplet breakup model, and (3) the ETAB (Enhanced TAB) secondary droplet breakup model. The choice between various models depends on the specific application. Further details of the atomization models and their assessment in several validation cases can be found in Refs. [3 \& 17 .

\subsection{SUPERHEAT SPRAY MODELING}

Flashing phenomena refers to a process that is in thermodynamic non-equilibrium when a liquid is superheated [42-43]. The reasons for its occurrence are mainly two-fold [42-43]: (1) a liquid fuel can be heated to a temperature above its saturation temperature, and (2) when a liquid is depressurized rapidly it can lead to flashing as the thermal inertia tends to maintain its bulk internal temperature above the saturation temperature. Although flash evaporation is considered to be detrimental to engine performance under normal circumstances, it can have some potential benefits. It is known to produce a fine spray with enhanced atomization, increase effective spray angle, and decrease spray penetration [44].

An understanding of flash injection is of importance in some applications involving scramjet and ramjet afterburners because the same liquid fuel is often used as a coolant. Also, the engine conditions are such that nozzles operate at low back pressures with supersonic outflow [44]. The objective of our work is to establish a baseline accuracy for existing atomization and vaporization models used in the prediction of a superheated spray by undertaking a critical review of existing experimental data available in the literature for validation. This work was funded through the supersonics (SUP) and subsonic fixed wing (SFW) project office initiatives on high altitude emissions of the NASA's fundamental aeronautics program. As a part of this investigation, we incorporated the following models into OpenNCC: (1) the superheat Vaporization Model of Zuo, Gomes, \& Rutland [45], and Schmehl \& Steelant [46-47], (2) Superheat Vaporization model of Lee et al. [48], \& (3) the flash-induced atomization model of of Lee et al. [48]. In the superheated regime, the thermo-physical liquid properties are replaced by the two-phase properties of a superheated fluid [48]. Further details of our superheat modeling approach can be found in [3 \& 18].

This modeling approach was applied in the validation of a flashing jet generated by the sudden release of pressurized R134A from a cylindrical nozzle [18]. Also, we investigated the differences between the superheat vaporization models of [45] \& [48] within the context of both reacting and non-reacting flow calculations of a Parker-Hannifin pressure swirl atomizer. In a separate investigation, we also looked into the impact of superheat conditions on the spray characteristics of a single-element LDI combustor [18].

\subsection{SUPERCRITICAL SPRAY MODELING}

There is a need for understanding droplet vaporization behavior at supercritical conditions because of the increasingly high operating pressures encountered in some gas-turbine combustors. The engine operating pressures can sometime exceed the supercritical pressure of liquid fuels such as jet-a. Because of its practical and fundamental importance, several experimental and theoretical investigations were undertaken to understand droplet gasification occurring at supercritical conditions [23-28 \& 33]. Most of the numerical investigations that appeared in the literature to study the vaporization behavior of an isolated single spherical droplet were based on the coupled twophase, unsteady Navier-Stokes equations in 1-D spherical coordinates [23-28]. However, it is noteworthy that the resulting gas-liquid interface analysis at the droplet surface becomes extremely complicated for a multi-component mixture. Also, the numerical solution is complicated by several factors as it needs to take into account the high-pressure corrections associated with various transport and thermo-physical properties. The supercritical droplet vaporization differs from low-pressure droplet vaporization models in several important ways. For example, the results of Zuo et al [27-28] showed that under low to moderate temperatures, first there would be an increase in droplet lifetime before it starts to fall 
off with an increase in pressure. Their results also showed that there would be a monotonic decrease in droplet lifetime with increase in pressure at high ambient temperatures.

However, none of these analyses could be directly incorporated into a comprehensive spray calculation because of any such detailed treatment would be prohibitively expensive in terms of the required computer CPU resources. Also, they fail to address how to handle the supercritical droplet vaporization in a real gas-turbine environment in which a droplet traverses through a non-stagnant gas. Since our main interest lies in implementing a viable high-pressure droplet vaporization model into our spray code, we attempted to incorporate some important aspects of high pressure modeling derived from Refs. [23-28] into the existing framework of our spray formulation. In what follows we describe some important aspects of supercritical droplet vaporization:

(1) The non-idealities in gas-phase become increasingly more pronounced as the pressure approaches a supercritical state. There are several widely used equations of state that provide accurate representation at high pressures, namely the PengRobinson (PR), Redlich-kwong (RK), and SoaveRedlich-Kwong (SRK). In our calculations, we implemented the PR EOS because of its simplicity and also known to provide accurate representation [2728]. Also, it is known to provide reasonable results when calculating the phase equilibrium at the droplet surface.

(2) At low pressure conditions, the solubility of the ambient gases in liquid phase can be neglected. The corresponding gas-phase composition at the droplet interface can be calculated by means of Raoult's law [3]. At high pressures, the phase equilibrium calculations at the droplet surface need to take into account fugacity of each individual species. More on how to evaluate the phase equilibrium at the droplet surface can be found in Section 2.5.1.

(3) Both liquid and gas compositions encountered in a gas turbine combustion device are truly multi-component in nature. However, all the numerical investigations reported so far on supercritical droplet vaporization are based on a binary mixture [23-28 \& 33]. Even for a binary mixture, the phase equilibrium calculations at high pressures present a formidable challenge. As it becomes very difficult to solve anything other than a binary mixture, we restrict the phase equilibrium calculations to a binary mixture involving a combination of a single- component surrogate fuel and nitrogen. Such a combination seemed to provide a reasonable representation for a fuel droplet vaporizing at high pressures [27-28].

(4) The transport properties in both liquid and gas phases become increasingly pressure dependent at high pressures. More on how we evaluate the transport properties at high pressures can be found in [3].

(5) As the droplet surface approaches a transcritical state, the latent heat of vaporization diminishes to zero. In our current calculations, the latent heat of vaporization is calculated by a formulation as described in Section 2.5.2.

(6) The solubility of the ambient gases in liquid phase becomes increasingly important at high pressures. However in our present calculation, we ignore to take into consideration the multi-component nature of droplet behavior for the following reasons: For gas turbine combustors of our interest, the gas pressure seldom exceeds more than twice the critical pressure of jet fuels. Within that pressure range, the liquid phase solubility of gas could be ignored since it could be shown from phase equilibrium calculations that the liquid-phase mass fraction of nitrogen remains less than three percent even when the droplet surface temperature reaches near critical temperature.

(7) Zhu et al. [27-28] studied the influence of gas-phase unsteadiness on droplet vaporization, and also quantified to some degree the resulting differences from the quasi-steady and transient models. Their results show that unsteadiness seemed to persist over a wide region during a brief early transient period after a droplet is suddenly introduced into an otherwise stagnant gas. After this initial transition period, some unsteadiness remains persistent in a small region closer to the droplet surface. Initially, the quasi-steady model seemed to produce a smaller regression rate when compared with the transient model. But in the later stages of droplet lifetime, it seemed to produce a much higher regression rate. The influence of unsteadiness seemed to increase with an increase in both ambient pressure and temperature. However in our present calculations, we expect for the quasi-steady model to provide a useful approximation for the following reasons: (1) We are interested mainly in gas pressures not too far above the critical pressure of the liquid fuel, and (2) Also, we are interested in a droplet moving a stratified gas where the unsteadiness associated with initial transient can be neglected. It is because our droplet 
models are based on what happens after the initial atomization phase. So the neglected influence of unsteadiness is mostly limited to a small region near the droplet surface.

\subsubsection{Equilibrium Relations Valid at High Pressure Conditions} isfy

At high pressures, the equilibrium needs to sat-

$$
\begin{gathered}
T^{L}=T^{V} \\
P^{L}=P^{V} \\
f_{i}^{L}=f_{i}^{V}=>x_{i} \phi_{i}^{L}=y_{i} \phi_{i}^{V}
\end{gathered}
$$

where $f_{i}$ is the fugacity of the ith species, $x_{i}$ is the mole fraction species in the liquid phase, $y_{i}$ is the mole fraction species in the vapor phase, and $\phi_{i}$ is the corresponding fugacity coefficient of the ith species which is given by

$\ln \phi_{i}=\frac{b_{i}}{b_{m}}(Z-1)-\ln \left(Z-B^{\star}\right)+\frac{A^{\star}}{2 \sqrt{2} B^{\star}}\left(\frac{b_{i}}{b_{m}}-\delta_{i}\right) \ln \frac{Z+B^{\star}(1+\sqrt{2})}{Z+B^{\star}(1-\sqrt{2})}$

where $\quad \frac{b_{i}}{b_{m}} \quad=\quad \frac{T_{c i} / P_{c i}}{\sum_{i} y_{j} T_{c j} / P_{c j}}, \quad$ and $\delta_{i}=\frac{2 a_{i}^{1 / 2}}{a_{m}} \sum_{i} x_{j} a_{j}^{1 / 2}\left(1-k_{i j}\right)$. There are two more additional equations that need to be satisfied at the droplet interface,

$$
\begin{aligned}
& \sum_{i} y_{i}=1 \\
& \sum_{i} x_{i}=1
\end{aligned}
$$

For a given $\mathrm{P}$ and $\mathrm{T}$ of a binary mixture, there are six equations and six unknowns: $Z_{v}, Z_{l}, y_{1}, y_{2}$, $x_{1}$, and $x_{2}$. The Peng-Robinson EOS yields solution for $Z_{v}$ and $Z_{l}$ and the other four unknowns are calculated from the solution of Eqs. (3) to (6). The solution for this highly non-linear set of equations is obtained from the use of a Newton-Raphson iterative method.

\subsubsection{Calculation of Latent Heat of Vaporization at High Pressure}

The enthalpy of the species can be calculated from

$$
h_{i}=h_{i}^{o}-R T^{2}\left[\frac{\partial \ln \phi_{i}}{\partial T}\right]_{p, x_{i}}
$$

The related derivatives in the above equation are given by

$$
\begin{aligned}
\frac{\partial \ln \phi_{i}}{\partial T}= & \frac{b_{i}}{b} \frac{\partial Z}{\partial T}-\frac{\left(\frac{\partial Z}{\partial T}+\frac{B^{\star}}{T}\right)}{Z-B^{\star}}-\frac{A^{\star}\left(\frac{b_{i}}{b}-\delta_{i}\right)\left(\frac{Z}{T}+\frac{\partial Z}{\partial T}\right)}{Z^{2}-B^{\star 2}+2 Z B^{\star}} \\
+ & \frac{1}{2 \sqrt{2} B^{\star}}\left(\frac{b_{i}}{b}-\delta_{i}\right)\left(\frac{\partial A^{\star}}{\partial T}+\frac{A^{\star}}{T}\right) \ln \frac{Z+B^{\star}(1+\sqrt{2})}{Z+B^{\star}(1-\sqrt{2})} \\
& -\frac{A^{\star}}{2 \sqrt{2} B^{\star}} \frac{\partial \delta_{i}}{\partial T} \ln \frac{Z+B^{\star}(1+\sqrt{2})}{Z+B^{\star}(1-\sqrt{2})} \\
\frac{\partial a_{i}^{1 / 2}}{\partial T}= & -\frac{R f_{w i}}{2}\left(\frac{0.45724 T_{c i}}{T P_{c i}}\right)^{1 / 2} \\
\frac{\partial a}{\partial T} & =-\frac{R}{2}\left(\frac{0.45724}{T}\right)^{1 / 2} \sum_{i} \sum_{j} y_{i} y_{j}\left(1-k_{i j}\right) \\
\frac{\partial A^{\star}}{\partial T} & =A^{\star}\left[\frac{1}{a} \frac{\partial a}{\partial T}-\frac{2}{T}\right] \\
\frac{\partial B^{\star}}{\partial T} & =-\frac{B}{T}
\end{aligned}
$$

$\frac{\partial Z}{\partial T}=$

$\frac{\left[-\frac{\partial A}{\partial T}\left(Z-B^{\star}\right)+\frac{B^{\star}}{T}\left[Z^{2}-2 Z\left(1+3 B^{\star}\right)-A^{\star}+2 B^{\star}+3 B^{\star 2}\right]\right.}{3 Z^{2}-2\left(1-B^{\star}\right) Z+A^{\star}-2 B^{\star}-3 B^{\star 2}}$

$\frac{\partial \delta_{i}}{\partial T}=$

$\delta_{i}\left[\frac{1}{a_{i}^{1 / 2}} \frac{\partial a_{i}^{1 / 2}}{\partial T}-\frac{1}{a} \frac{\partial a}{\partial T}\right]+2 \frac{a_{i}^{1 / 2}}{a} \sum_{j} y_{j}\left(1-k_{i j}\right) \frac{\partial a_{i}^{1 / 2}}{\partial T}$

Finally, once the enthalpies in both phases are known, the latent heat of vaporization is simply calculated as follows

$$
L_{i}=h_{i}^{v}-h_{i}^{l}
$$

\subsection{COMPUTATIONAL BOUNDARY CONDITIONS: WALL, PERIODIC, INFLOW, \& EXIT}


The spray code supports all of the boundary conditions that exist in the current version of the OpenNCC CFD module. First, we are going to provide a summary of the droplet and wall interaction models. There are several possible outcomes following a droplet impact with a wall. They can be broadly classified into four categories: (1) the droplet may move along a wall after its impact but still keeps on vaporizing, (2) the droplet may rebound after its impact with the wall, (3) the droplet may stick to the wall after its impact but keeps on vaporizing, or (4) the droplet may be shattered after its impact with the wall. The first outcome is the default boundary condition that was originally implemented into our code. It is based on the assumption that a droplet, after having lost most of its momentum upon impingement with the walls, moves along the wall surface with a velocity closer to that of the surrounding gas-film. The last three outcomes are based on the models described in Refs. [49-50]. They are implemented partially based on the coding received from CFDRC.

In order to implement the droplet and wall interaction models, there is a need to determine the intersection location of a particle crossing a wall, $\underline{x}_{b l, k}$. It can be determined based on the the known initial particle location, $\underline{x}_{i k}$, particle velocity, $\underline{V}_{i k}$, and other geometrical considerations of the grid cell. Based on vector analysis, we have determined it as follows

$\underline{x}_{b l, k}=\left(x_{i k}+u^{\star} u_{i n k}, y_{i k}+u^{\star} v_{i n k}, z_{i k}+u^{\star} w_{i n k}\right)$

where

$$
u^{\star}=\frac{\underline{a}_{n} \cdot\left(\underline{x}_{c w f}-\underline{x}_{i k}\right)}{\underline{a}_{n} \cdot \underline{V}_{i n k}},
$$

$\underline{V}_{i n k}$, is the velocity normal $\left(=\underline{V}_{i k} /\left|\underline{V}_{i k}\right|\right)$, and $\underline{x}_{c w f}$ is the center location of the wall face of the grid cell. In what follows, we define various variables Reynolds number, $R e_{s w, k}$, Weber number, $W e_{s w, k}$, Ohnesorge number, $O h_{s w, k}$, surface energy, $E_{s w}$, and viscous dissipation, $V_{d i s}$ - used in the spray-wall inraction modeling.

$$
\begin{aligned}
R e_{s w, k} & =\frac{2 \rho_{k} r_{k}\left|\underline{V}_{i k}\right|}{\mu_{k}} \\
W e_{s w, k} & =\frac{2 \rho_{k} r_{k}\left|\underline{V}_{i k}\right|^{2}}{\sigma_{k}}
\end{aligned}
$$

$$
\begin{gathered}
O h_{s w, k}=\frac{\sqrt{W e_{s w, k}}}{R e_{s w, k}} \\
E_{s w}=\pi \sigma_{k}\left(2 r_{k} \beta_{\max }\right)^{2}(1-\cos \alpha) / 4
\end{gathered}
$$

where

$$
\beta_{\max }=\frac{\sqrt{12+W e_{s w, k}}}{3(1-\cos \alpha)}+\frac{4 W e_{s w, k}}{\sqrt{R e_{s w, k}}},
$$

the droplet impact angle, $\alpha$, is given by

$$
\alpha=\frac{\pi}{2}-\cos ^{-1} \gamma,
$$

and the droplet frequency, $\gamma$, is

$$
\gamma=\frac{\left(\underline{x}_{b l, k}-\underline{x}_{i k}\right) \cdot \underline{a}_{n}}{2 r_{k}},
$$

and

$$
V_{d i s}=\frac{2 \pi r_{k} \rho_{k}\left|\underline{V}_{i k}\right|^{2}\left(2 r_{k} \beta_{\max }\right)^{2}}{3 \sqrt{R e_{s w, k}}}
$$

The droplet outcome after the interaction with the wall is determined based on the following criteria:

\section{Shattering Droplet:}

A droplet shatters upon impact with a wall when $W e_{s w, k}>W e_{c r i t}$, where the critical Weber number is defined as follows

$$
W e_{\text {crit }}=9.9^{10} O h^{2.8}
$$

The average size of the shattered droplet size after impact is given by

$$
r_{\text {ave,shat }}=\max \left(r_{l 1, \text { shat }}, r_{l 2, \text { shat }}\right)
$$

where $r_{l 1, \text { shat }}$ and $r_{l 2, \text { shat }}$ represent the two limits of droplet sizes as determined by

$$
\begin{aligned}
r_{l 1, \text { shat }} & =\frac{\sigma_{k} W e_{c r i t}}{2 \rho_{k}\left|\underline{V}_{i k}\right|^{2}} \\
r_{l, \text { shat }} & =\frac{4 \pi r_{k}^{3} \sigma_{k}}{E_{s w}}
\end{aligned}
$$

Once $r_{\text {ave,shat }}$ is determined, the total number of shattered droplets produced is given by 


$$
N_{\text {shat }}=\operatorname{int}\left[\left(\frac{r_{k}}{r_{\text {ave }, \text { shat }}}\right)^{3}\right]
$$

However, it is important to note that the number of shattered droplets generated is limited to 2 as shown below

$$
N_{\text {shat }}=\min \left(2, \operatorname{int}\left[\left(\frac{r_{k}}{r_{\text {ave }, \text { shat }}}\right)^{3}\right]\right)
$$

The actual size of the shattered droplets is calculated by introducing some randomness as shown below

$$
r_{\text {shat }}=r_{\text {ave }, \text { shat }}(0.85+0.30 R N D)
$$

where RND is a random number between $(0,1)$. The droplet position and velocity of the shattered droplets is determined by the following criteria.

Upon impact with a wall, a shattered droplet emerges from the wall in a direction determined by the modified reflection normal vector, $\underline{x}_{r n k}$.

$$
\underline{x}_{r n k}=2(0.5-R N D)\left(\underline{x}_{i n k}-C_{r e s}\left(\underline{x}_{i n k} \cdot \underline{a}_{n}\right) \underline{a}_{n}\right)
$$

where $C_{r e s}$ stands for coefficient of restitution. Its value depends on the properties of the wall but has a value of 2 under normal reflecting conditions. Also, RND is added to introduce some randomness into the direction of reflection vector. Once $\underline{x}_{r n k}$ is determined, the new droplet position and velocity are determined.

$$
\begin{gathered}
\text { If } \underline{x}_{r n k} \cdot \underline{a}_{n}>0, \\
\underline{V}_{n k}=\left|\underline{V}_{i k}\right| \underline{x}_{r n k} \\
\underline{x}_{n k}=\underline{x}_{b l, k}+\left|\underline{x}_{b l, k}-\underline{x}_{i k}\right| \underline{x}_{r n k}+C_{o f f} \underline{a}_{n}
\end{gathered}
$$

where $C_{o f f}$ stands for offset and is usually assigned a tiny value but in our present calculations it is assigned a value of $0.01\left|x_{c w f}-x_{c g c}\right|$, where $x_{c g c}$ is the center location of grid cell.

$$
\begin{gathered}
\text { but if } \underline{x}_{r n k} \cdot \underline{a}_{n} \leq 0, \\
\underline{V}_{n k}=10^{-06} \underline{a}_{n} \\
\underline{x}_{n k}=\underline{x}_{b l, k}+C_{o f f} \underline{a}_{n}
\end{gathered}
$$

Once the droplet size, location, and velocity of the shattered droplets are determined, the rest of the droplet properties can easily be deduced from the initial conditions of incident droplet.

\section{Sticking Droplet to the Wall:}

A droplet sticks to the wall when $E_{s w}>V_{d i s}$. After being stuck to the wall, the impacted droplet size remains the same but continue to vaporize in time. The droplet velocity and position are assigned the following values:

$$
\begin{gathered}
\underline{V}_{n k}=10^{-06} \underline{a}_{n} \\
\underline{x}_{n k}=\underline{x}_{b l, k}+C_{o f f} \underline{a}_{n}
\end{gathered}
$$

The rest of the droplet properties are deduced from the initial conditions of incident droplet.

\section{Rebounding Droplet:}

If none of the above conditions are met for the outcomes of either droplet shattering or droplet sticking to a wall, the droplet is assumed to rebound after its impact with a wall. After the rebound, the droplet size remains intact as in the incident droplet. Both the droplet velocity and position are calculated as in the droplet shattering but randomness is removed from the reflection vector. So it is calculated as in Eqs. (31-34) but RND is assigned a value of zero. The rest of the droplet properties are deduced from the initial conditions of incident droplet.

\section{Moving Droplet Along the Wall:}

When this option is invoked, a droplet is assumed to move along next to the wall surface after its subsequent impact with a wall. Such a droplet would experience no change in its size after its impact but it is allowed to keep on vaporizing as it moves along the wall surface. The droplet velocity and position are calculated as follows

$$
\begin{gathered}
\underline{V}_{n k}=\underline{V}_{g s} \\
\underline{x}_{n k}=\underline{x}_{b l, k}+C_{o f f} \underline{a}_{n}
\end{gathered}
$$

where $\underline{V}_{g s}$ is the velocity of the the surrounding gas.

Finally, in what follows we highlight how some other boundary conditions are implemented: 
- The implementation of the periodic boundary conditions becomes rather complicated. It is because one needs to keep track of a particle leaving the computational domain from one periodic boundary, and for every particle that leaves the domain, a second particle reenters the computational domain through a corresponding second periodic boundary. Also, one needs to take into account the possibility of the two computational cells where the particles leave and reenter the domain being assigned to a different processor. We incorporated the periodic implementation as a part of the particle search algorithm. It is important to note that the boundary conditions are implemented with the help of some appropriately defined transformation matrices. Also, the particle velocities of the entering particle need to be adjusted accordingly based on the orientation of respective periodic boundaries.

- The symmetric boundary condition is implemented in such a way to satisfy the criterion that for every particle crossing the symmetry line, a similar one re-enters the domain in a direction determined by the reflection vector.

- When the particles move out of the exit boundary, they are taken out of the computation.

\subsection{DESCRIPTION OF INITIAL SPRAY CONDITIONS}

The spray computations facilitate the use of multiple fuel injectors. The same or a different type of liquid fuel can be specified for each one of different injectors. The initial droplet temperature is assumed to be the same for all different droplet groups of a given injector. The liquid fuel injection is simulated by introducing a number of discretized parcels of liquid mass at the beginning of every fuel-injection time step, $\Delta t_{i l}$.

The initial droplet distribution for a given injector could be specified by making use of one of the three available options: (1) by providing a complete specification of the initial conditions by means of a spray table, (2) by means of some available correlations, or (3) by means of some available primary atomization models.

\section{RESULTS FOR A SINGLE-ELEMENT LDI WITH AXIAL $60^{\circ}$-VANE SWIRLER}

\author{
3.1 DETAILS OF THE COMPUTATIONAL GRID \\ \& CFD MODELS
}

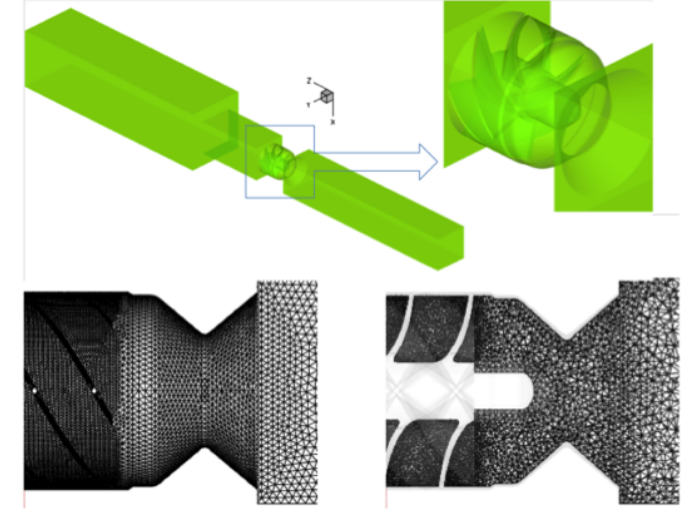

Fig. 1 Computational geometry and mesh for the single-element LDI with $60^{\circ}$ axial-swirl vanes (courtesy of Kumud Ajmani).

The computational geometry and grid for the single element LDI-1 configuration are shown in Fig. 1. The computational grid was furnished to us by Kumud Ajmani at NASA GRC. It comprises of an axial swirler with six $60^{\circ}$ blade-passages for air flow. It is placed in a converging-diverging Venturi downstream of the combustion section [21]. The liquid fuel is injected from a nozzle located near the center of the air-swirler exit. The exit is located at or slightly upstream of the throat.

More details on the computational grid can be found in Ref. [21]. The grid mesh comprises of 1.1 million tetrahedral elements. It satisfies the following criteria: (1) it ensures that the calculated pressure drop for a given air mass flow rate be predicted within $10 \%$ of the experimental value, (2) it ensures that proper pressure drop is maintained across all the swirl vane-passages by providing sufficient grid clustering upstream of the swirler and by providing sufficient and yet uniform grid spacing along each of the swirl air-passages, and (3) close attention was paid to the grid clustering and stretching in the combustion region in order to properly resolve all the wallboundary and shear-layer regions [22].

The present calculation is mainly undertaken to demonstrate the applicability of the newly implemented high-pressure spray models in conjunction with the high-pressure EOS. More details on the liquid-phase computations can be found in Sec. 2 .

The gas-phase computations are based on OpenNCC RANS with standard two-equation turbulence model, the Peng-Robinson EOS, \& a 14-species \& 18-step reduced chemical kinetics mechanism of 
Table 1. Liquid spray initial test conditions.

\begin{tabular}{|c|c|c|c|}
\hline fuel/air ratio & $\phi$ & mass flow rate, $\mathrm{kg} / \mathrm{s}$ & droplet velocity, $\mathrm{m} / \mathrm{s}$ \\
\hline 0.016 & 0.24 & 0.002029 & 21.17 \\
\hline 0.029 & 0.43 & 0.003720 & 38.64 \\
\hline 0.038 & 0.55 & 0.004820 & 50.64 \\
\hline
\end{tabular}

[21]. The reduced kinetics mechanism was optimized to match the emission predictions of a detailed JetA combustion chemical kinetics mechanism over a wide range of equivalence ratios $(0.1<\phi<1.5)$. The gas-phase computations are performed based on a central-differencing scheme with second-order accurate discretization for both viscous and inviscid fluxes. It makes use of a Jameson operator based on blend of second and fourth-order artificial dissipation terms to achieve numerical stability. The steadystate RANS solution is obtained by making use of an explicit, four-stage Runge-Kutta scheme. The convergence to steady-state is accelerated by making use of residual smoothing of residuals in pseudo time. It is noteworthy that no attempt was made in these calculations to include any chemistry-turbulence models.

\subsection{INITIAL TEST CONDITIONS}

The air enters the combustion chamber at a mass flow rate of $0.12683 \mathrm{~kg} / \mathrm{s}$, gas temperature 811.1 deg. $\mathrm{K}\left(T_{3}\right)$, and an upstream pressure of $27.6 \mathrm{~atm}$. However, instead of specifying the pressure at the inflow, the inflow pressure was allowed to float. Its value was determined based on a combination of boundary conditions specified at the exit and inflow boundaries. The air mass flow rate was specified at the inflow and a uniform back pressure at the exit boundary. The back pressure is adjusted until the upstream pressure matches the desired value. Based on the numerical experiments performed by [22] the specified value for the fixed static pressure at the exit boundary was determined to be $97 \%$ of $P_{3}$. The steady-state OpenNCC RANS solution predicted the pressure drop to be within $10 \%$ of the measured experimental value [22].

The location of the fuel element based on a Woodward FT design is shown in Fig. 1. The liquid fuel used in the experiment was Jet-A. Table 1 shows the initial test conditions used in three different calculations. The table contains the fuel/air ratio, the

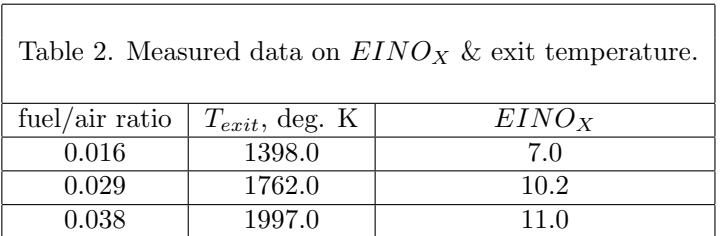

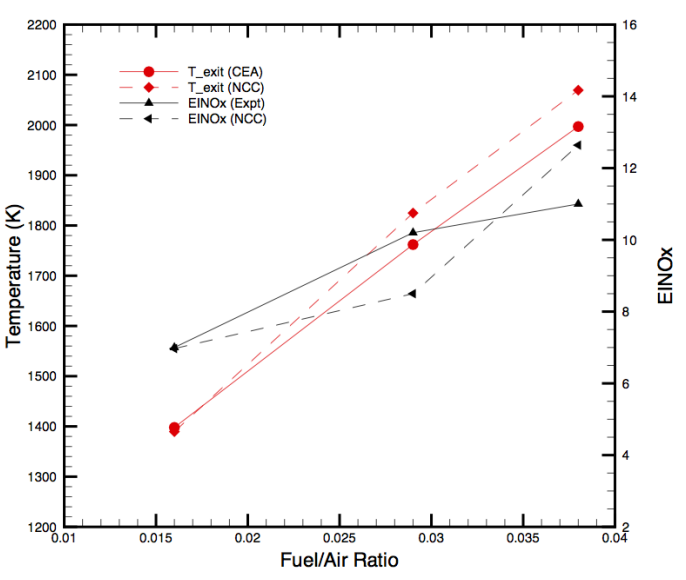

Fig. 2 Comparisons of exit temperature \& $E I N O_{X}$ versus experimental data.

corresponding overall equivalence ratio $(\phi)$, the liquid mass flow rate, \& initial droplet velocity. These test conditions were chosen to cover emissions predictions over a wide range of $\phi$. Based on the input received from Woodward Ft, the initial droplet distribution function was determined based on a correlation with a Sauter mean diameter (SMD) of 8.8 microns [3]. A single-component surrogate fuel of $C_{11} H_{21}$ was chosen to model jet-a. The critical pressure of $C_{11} H_{21}$ is $1.82 \mathrm{mPa}$ and its critical temperature, $658.2 \mathrm{deg}$. $\mathrm{K}$. The initial liquid temperature was specified to be $450 \mathrm{~K}$.

\subsection{RESULTS FOR LDI-1, 27ATM}

The reported experimental data for $E_{I N O_{X}}$ and $T_{\text {exit }}$ for different fuel/air ratios are shown in 


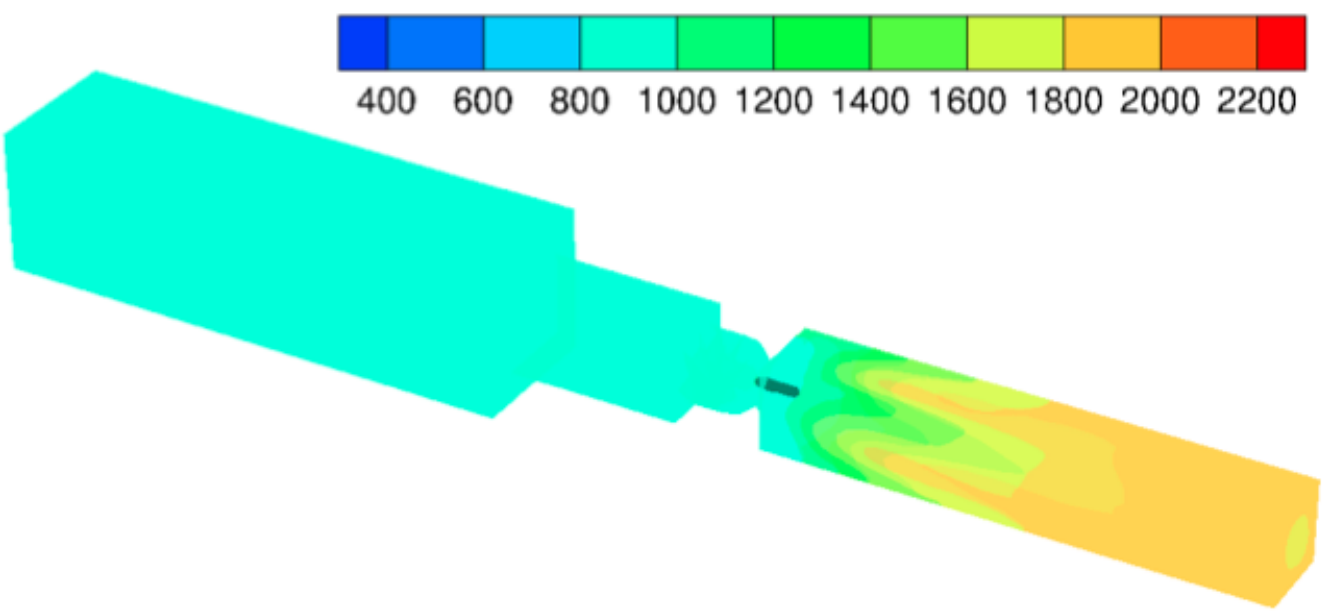

Fig. 3 A 3D perspective view of temperature (deg. K) contours \& spray particle distribution.

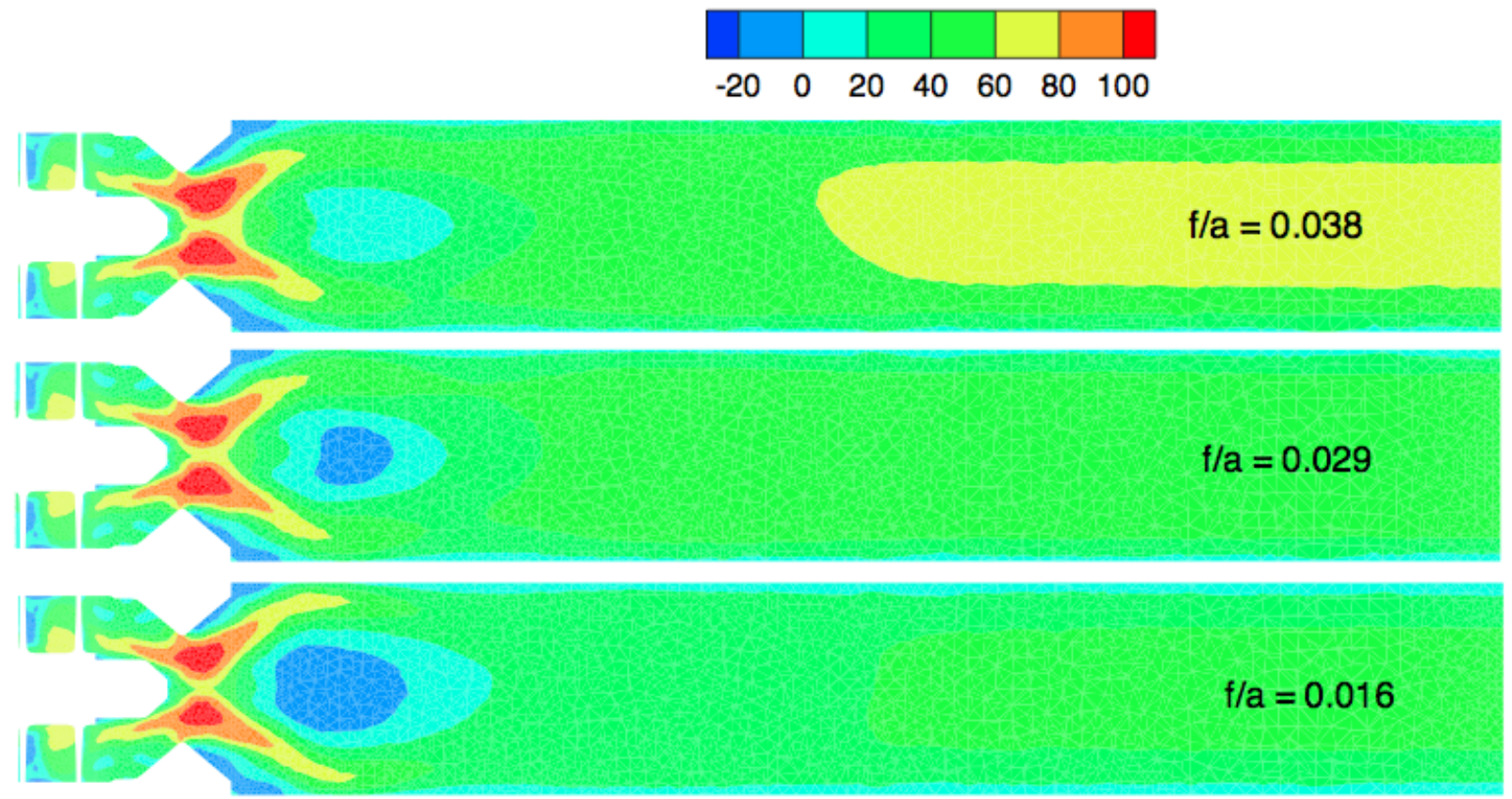

Fig. 4 Axial velocity $(\mathrm{m} / \mathrm{s})$ contours at the $\mathrm{Y}=0$ plane. 


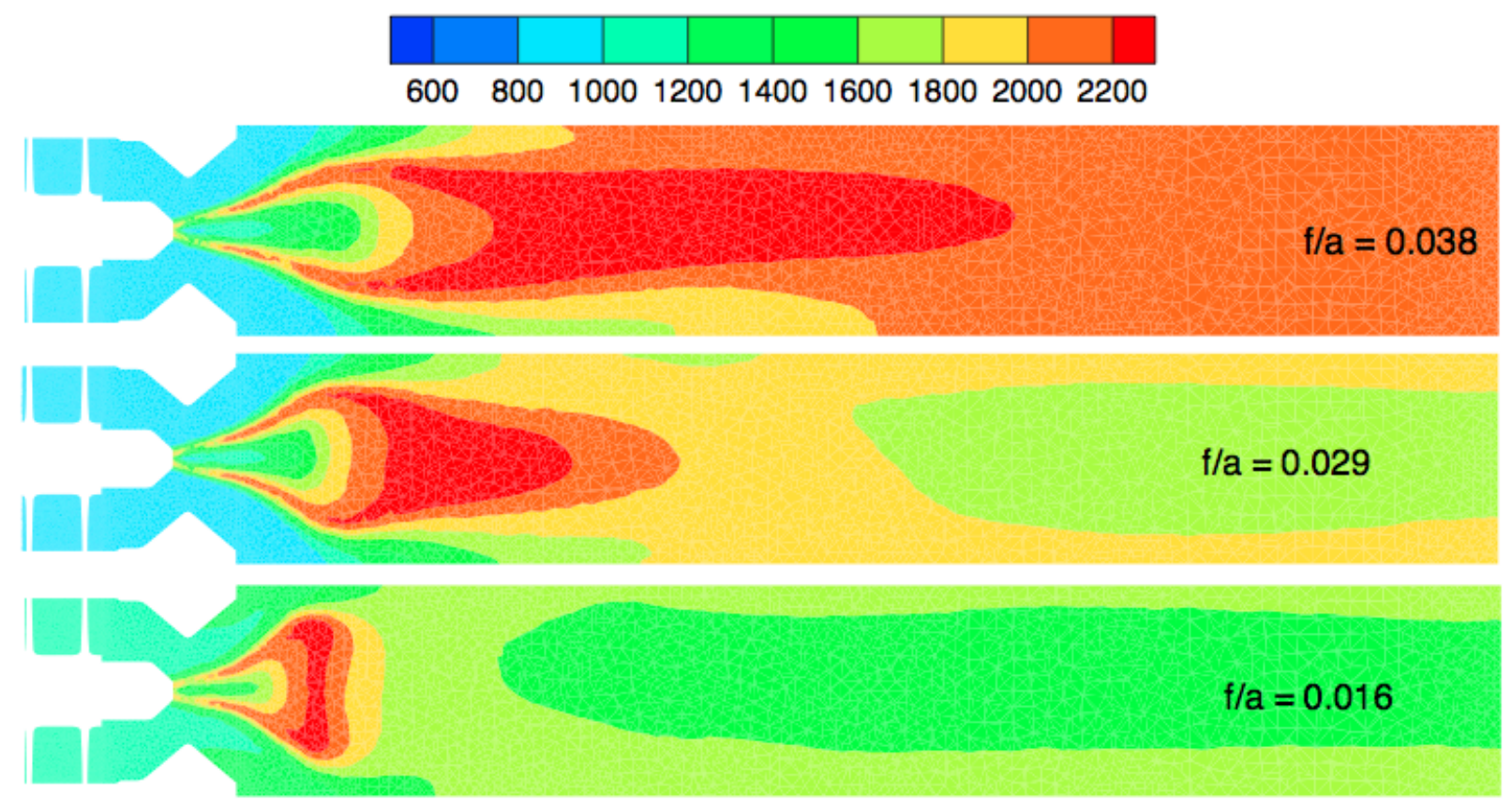

Fig. 5 Temperature (deg. $\mathrm{K})$ contours at the $\mathrm{Y}=0$ plane.

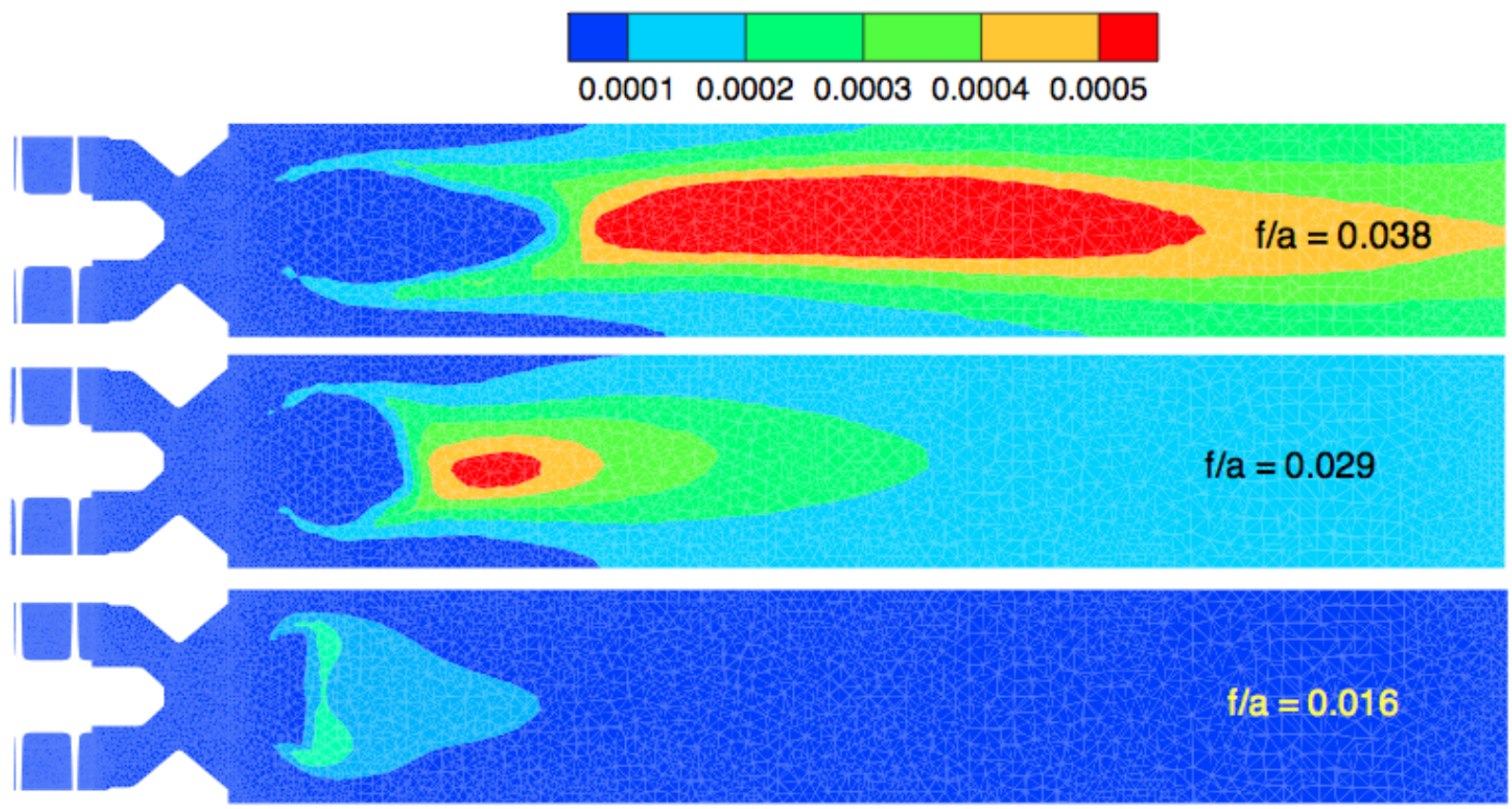

Fig. $6 N O_{X}$ contours at the $\mathbf{Y}=\mathbf{0}$ plane. 
Table 2. Fig. 2 shows the comparisons of $E I N O_{X}$ and $T_{\text {exit }}$ versus $\mathrm{F} / \mathrm{A}$ ratio. There is a good comparison for both $T_{\text {exit }}$ and $E I N O_{X}$. The calculations are consistent with the experimental data showing an increase in exit temperature with an increase in $\mathrm{F} / \mathrm{A}$ ratio. Also, the attendant increase in $E I N O_{X}$ due to a rise in overall high temperature region with an increase in $\mathrm{F} / \mathrm{A}$ ratio is clearly evident.

Fig. 3 shows the 3D perspective view of the temperature contours together with the spray particle distribution. The temperatures range between 450 to 2200 deg. K. The flame originates downstream of the spray and is primarily supported by the swirl-induced primary recirculation. The high temperature region is located downstream of this primary recirculation in the middle of the combustion chamber.

The axial velocity contours (at the $Y=0$ plane) from the three calculations are shown in Fig. 4. The values range from -20 to $100 \mathrm{~m} / \mathrm{s}$. The magnitude of the velocity is the largest near the throat, Its effect is more magnified by the expansion of the reacting gas. The flow field is mainly characterized by a swirlinduced primary recirculation formed in the central region downstream of of the nozzle and a corner vortex formed on the diverging section of Venturi near the wall. The negative velocities of the primary recirculation are more pronounced toward the lower end of the F/A ratio. The results are consistent with what was observed experimentally in [20]. The corresponding temperature contours at the $Y=0$ plane are shown in Fig. 5. The temperatures range between 450 to $2200 \mathrm{deg}$. K. The lowest temperature is found to occur near the evaporating region of the spray region where the droplets enter the flow field with an initial temperature of $450 \mathrm{deg}$. K. The flame is primarily supported by the primary recirculation causing the formation of a high temperature region downstream. An increase in the exit temperature is clearly evident with an increase in the $\mathrm{F} / \mathrm{A}$ ratio. Finally, the corresponding $N O_{X}$ contours at the $Y=0$ plane are shown in Fig. 6 . The range varies between 0.0 to 0.025 . As expected, the location of peak $N O_{X}$ lies closer to the high temperature region. Also, an increase in $N O_{X}$ is clearly evident with an increase in $\mathrm{F} / \mathrm{A}$ ratio. It is noteworthy that the qualitative nature of the predicted results is consistent with the experimental results of [20].

\section{CONCLUDING REMARKS}

In this paper, we summarized some main capabilities (\& features) of the spray module, LSPRAY-V. which was developed as a part of the national combustion code development over the last two decades. It facilitates the calculation of multicomponent liquid sprays over a wide range of gas-turbine engine operating conditions. Its use has been demonstrated in the numerical investigation of various reacting/nonreacting flows encountered in gas-turbine combustors, stratified-charge rotary combustion (Wankel) engines, supersonic and pulse detonation combustion devices [1-3, \& 9-22].

The paper also presented some results obtained from the application of this spray modeling approach to a high pressure reacting spray generated by a single-element LDI-1 combustor with $60^{\circ}$ axial-swirl vanes. The solution was based on OpenNCC RANS with a high-pressure EOS, a high-pressure droplet vaporization model, and an optimized emissions reduced chemical kinetics mechanism. The results provided reasonable agreement with the available experimental data for both $T_{\text {exit }}$ and $E I N O_{X}$ over a wide range of fuel/air mixure ratios.

\section{ACKNOWLEDGEMENT}

This work was supported by NASA with Dr. Jeff Moder acting as the technical monitor. The author would like to extend his sincere appreciation to him for his many valuable contributions to the spray code, Dr. Thomas Wey for his help with OpenNCC, and Dr. Kumud Ajmani for providing the grid used in the calculations.

\section{REFERENCES}

1. Liu, N.-S., Shih, T.-H., \& Wey, C.T., "Comprehensive Combustion Modeling and Simulation: Recent Progress at NASA Glenn Research Center," ISABE-2007-1268, 18th International Symposium on Air Breathing Engines, Beijing, China, September 2-7, 2007.

2. Liu, N.-S., \& Wey, C.T., "Updates to Simulation of a Single-Element Lean-Direct Injection Combustor Using a Polyhedral Mesh Derived From Hanging-Node Elements," NASA/TM2014-218108, Glenn Research Center, Cleveland, Ohio.

3. Raju, M.S., "LSPRAY-V: A Lagrangian Spray Module," NASA/CR-2015-218918, NASA Glenn Research Center, Cleveland, Ohio, November, 2015. 
4. W.A. Sirignano, "Fluid Dynamics of Sprays," Journal of Fluids Engineering, vol. 115, no. 3, pp. 345-378, September 1993.

5. Crowe, C.T., "Numerical Models for Dilute GasParticle Flows," Journal of Fluids, Vol. 104, pp. 297-301, 1982.

6. C.T. Crowe, M.P. Sharma, and D.E. Stock, The Particle-Source-in Cell (PSI-CELL) Model for Gas-Droplet Flows, J. Fluids Eng., vol. 99, pp. 325, 1977.

7. Y. El Banhawy and J.H. Whitelaw, Calculation of the Flow Properties of a Confined KeroseneSpray Flame, AIAA J., vol. 18, no. 12, pp. 1503-1510, 1980.

8. Faeth, G.M., "Mixing, transport, and Combustion in Sprays," Progress Energy Combustion Science, Vol. 13, pp. 293-345, 1987.

9. Raju, M.S., and Sirignano, W.A., "Spray Computations in a Centerbody Combustor," Proceedings of the 1987 ASME-JSME Thermal Engineering Joint Conference, Vol. 1, pp. 61-71, Honolulu, HI, March 1987. Also see Journal of Engineering for Gas Turbines and Power, Vol. 1, No. 4, pp. 710-718, October 1989.

10. Raju M.S., and Sirignano, W.A., "MultiComponent Spray Computations in a Modified Centerbody Combustor," Journal of Propulsion and Power, Vol. 6, No. 2, March-April 1990.

11. Raju, M.S., "AGNI-3D: A Computer Code for the Three-Dimensional Modeling of a Wankel Engine," Computers in Engine Technology, Proceedings IMechE, London, United Kingdom, pp. 27-37, 1991.

12. Raju, M.S., "Heat Transfer and Performance Characteristics of a Dual-Ignition Wankel Engine," Journal of Engines, the 1992 SAE Transactions, Section 3, pp. 466-509.

13. M.S. Raju, Application of Scalar Monte Carlo Probability Density Function Method For Turbulent Spray Flames, Numerical Heat Transfer, Part A, vol. 30, pp. 753-777, 1996.

14. M.S. Raju, Current Status of the Use of Parallel Computing in Turbulent Reacting Flows: Computations Involving Sprays, Scalar Monte
Carlo Probability Density Function \& Unstructured Grids, Advances in Numerical Heat Transfer, vol. 2, ch. 8, pp.259-287, 2000.

15. M.S. Raju, Scalar Monte Carlo PDF Computations of Spray Flames on Unstructured Grids With Parallel Computing, Numerical Heat Transfer, Part B, No. 2, Vol. 35, pp. 185-209, March 1999.

16. M.S. Raju, On the Importance of Chemistry/Turbulence Interactions in Spray Computations, Numerical Heat Transfer, Part B: Fundamentals, No. 5, Vol. 41, pp. 409-432, 2002.

17. Raju, M.S., \& Bulzan, D., "Assessment of Some Atomization Models used in Spray Calculations," ICCES1120110131487, Nanjing, China, 18-21 April, 2011, also as NASA/TM-2011217029, Glenn Research Center, Cleveland, Ohio, March 2011.

18. Raju, M.S., \& Bulzan, D., Current Status of Superheat Spray Modeling With NCC," AIAA 2012-3707, 48th Joint Propulsion Conference and Exhibit cosponsored by AIAA, ASME, SAE, and ASEE, Atlanta, Georgia, July 30August 1, 2012, also as NASA/TM2012-217295, Glenn Research Center, Cleveland, Ohio, March 2012.

19. M.S. Raju, "EUPDF-II - An Eulerian-Based Monte Carlo Probability Density Function (PDF) Solver - User's Manual," NASA/CR2004-213073, NASA Lewis Research Center, Cleveland, Ohio.

20. Tacina,R., Lee, P., \& Wey, C.W., "A LeanDirect-Injection Combustor Using a 9 Point Swirl-Venturi Fuel Injector," ISABE-2005-1106, 2005.

21. Ajmani, K., Kundu, K., \& Yungster, S.J., "Assessment of Reduced Mechanisms for Combustion ofJet Fuel in CFD Applications," Vol. 4, no. 2946, 50th AIAA/ASME/SAE/ASEE Joint Propulsion Conference, Cleveland,Ohio, July 2830, 2014.

22. Ajmani, K., Mongia, H., \& Lee, P., "Best Practices to Predict $\mathrm{NO}_{X}, \mathrm{CO}$, and Lean Blowout For Combustor Design," GT 2013-95669, Proceedings of ASME Turbo Expo 2013. 
23. Bellan, J., "Supercritical (and Subcritical) fluid Behavior and Modelling: Drops, Streams, Shear and Mixing Layers, Jets, and sprays," Prog. Energy Combust. Sci., Vol. 26, pp. 329-366, 2000.

24. Yang, V., "Modeling of Supercritical Vaporization, Mixing, and Combustion Processes in Liquid-Fueled Propulsion Systems," Proceedings of the Combustion Institute, vol. 28, pp. 925942,2000 .

25. Delplanque, J.-P., Sirignano, W.A., "Numerical study of the transient vaporization of an oxygen droplet at sub- and super-critical conditions," Int. J. of Heat and Mass Transfer, Vol. 36, No. 2, pp. 303314, 1993.

26. Gilver, S.D., and Agraham, J., " Supercirtical Droplet Vaporization and Combustion Studies," Prog. Energy Combust. Sci., Vol. 22, pp. 183197, 1996.

27. Zhu, G., Reitz, R.D., and Aggarwal, S.K., "GasPhase Unsteadiness and Its Influence on Droplet vaporization in Sub- and Super-Critical Environments," Int. J. of Heat \& Mass Transfer, Vol. 44, no. 16, pp. 3081-3093, 2001.

28. Zhu, G., and Aggarwal, S.K., "Fuel Droplet Evaporation in a Supercritical Environment," Transactions of the ASME-J. of Engineering for Gas Turbines and Power, Vol. 124, October, pp. 762-770, 2002.

29. Reid, R.C., Prausnitz, J.M., and Poling, B.E., "The Properties of Gases and Liquids," 4th Edition, McGraw-Hill Book Company, 1987.

30. Prausnitz, J.M., and Chueh, P.L., "Computer Calculation For High pressure Vapor-Liquid Equilibria," Prentice-Hall, Englewood Cliffs, N.J., 1968.

31. Peng, D.Y., and Robinson, D.B., Ind. Eng. Chem. Fundam., Vol. 15, pp. 59, 1976.

32. Aggarwal, S.K., Shu, Z., Mongia, H., and Hura, H., "Multicomponent Fuel Effects on the Vaporization of a Surrogate Single-Component Fuel Droplet," AIAA Paper 98-0157, 36th Aerospace Sciences Meeting, Reno, Nevada, Jan. 12-15, 1998.
33. Aggarwal, S.K., Shu, Z., Mongia, H., and Hura, H., "Multicomponent Single-Component Fuel Droplet Evaporation Under High Pressure Conditions," 34th AIAA/ASME/SAE/ASEE Joint Propulsion Conference \& Exhibit, Cleveland, OH, July 13-15, 1998.

34. Reichenberg, D., "The Viscosities of Gases at High Pressures," Natl. Eng. Lab., Rept. Chem. 38, East Kilbride, Glascow, Scotland, August 1975.

35. Stiel, L.I., and Thodos, G., AICHE J., Vol. 10, pp. 26, 1964.

36. Takahashi, S., J. Chem. Eng. Japan, Vol.7, pp. $417,1974$.

37. Reitz, R.D., \& Bracco, F.V., "Mechanism of Atomization of a Liquid Jet," Phy. Fluids, Vol. 25, ch. 10, Oct., 1982.

38. Tanner, F.X., "Liquid Jet Atomization and Droplet Breakup Modeling of Non-evaporating Diesel Fuel sprays," SAE Technical Paper 970050, 1998. Also SAE 1997 Transactions: Journal of Engines, Vol. 106, Sec. 3, pp. 127140, 1998.

39. Tanner, F.X., "A Cascade Atomization and Drop Breakup Model for the Simulation of HighPressure Liquid Jets," SAE Paper 2003-01-1044, 2003.

40. Reitz, R.D., "Modeling Atomization Processes in High-Pressure vaporizing Sprays," Atomization and Spray Technology, Vol. 3, pp. 309-337, 1987.

41. Reitz, R.D., and Diwakar, R. "Structure of HighPressure Fuel Sprays," SAE paper 870598, 1987.

42. Yildiz, D., Rambaud, P., Van Beeck, J., Buchlin, J.-M., "Characterization of Superheated Liquid Jet Atomization Phase Doppler Anemometer (PDA) and High-Speed Imaging," Proceedings of FEDSM2006: 2006 ASME Joint U.S.European Fluids Engineering Summer Meeting, July 17-20, 2006, Miami, Florida.

43. Yildiz, D., Rambaud, P., Van Beeck, J., Buchlin, J.-M., "Evolution of the Spray Characteristics in Superheated Liquid Jet Atomization in Function of Initial Flow Conditions," ICLASS-2006, Paper ID ICLASS06-122, Aug. 27-Sept 1, 2006, Kyoto, Japan. 
44. Lucas, K.D., Tseng, C.C., Pourpoint, T.L., Lucht, R.P., and Anderson, W.E., "Imaging Flashing Injection of Acetone at Jet Engine Augmentor Conditions," AIAA Paper 2007-1182, 45th AIAA Aerospace Sciences Meeting and Exhibit, Reno, Nevada, Jan. 8-11, 2007.

45. Zuo, B., Gomes, A.M., and Rutland, C.J., "Modeling Superheated Fuel Sprays and Vaporization," Inj. J. Engine Research, vol. 1, no. 4, pp. $321-336$.

46. Schmehl, R, and Steelant, J., "Flash-Evaporation of Oxidizer During Start-Up of an Upper-Stage Rocket Engine," AIAA Paper 2003-5075, 39th AIAA/ASME SAE/ASEE Joint Propulsion Conference and Exhibit, Huntsville, Alabama, July 20-23 2003.

47. Schmehl, R, and Steelant, J., "Evaluation of Oxidizer Temperature Drop in a Combustion Chamber," 4th International Conference on Launcher Technology "Space Launcher Liquid Propulsion, Liege, Belgium, December 3-6 2002.

48. Lee J., Fotache C., Gopalakrishnan, S., \& Schmidt, D., "Validated tools for Low Emissions Injector Design Using Superheated/Supercritical Fuels," NASA/CR-Year-2010/NNC07CB05C, Glenn Research Center, Cleveland, Ohio.

49. DesJardin, P.E., Presser, C., \& Disimile, P.J., " A Phenomenological Droplet Impact Model for Lagrangian Spray Transport", AIAA 2003-1322, AIAA 41st Aerospace Sciences Meeting and Exhibit, Reno NV, January 6-9, 2003.

50. Stanton, D.W., \& Rutland, C.J., "MultiDimensional Modeling of Thin Liquid Films and Spray-Wall Interactions Resulting From Impinging Sprays", Intl. J. Heat and Mass Transfer, Vol. 41, pp. 3037-3054, 1998. 